\title{
Prevalence of malaria parasitaemia among asymptomatic women at booking visit in a tertiary hospital, North- central Nigeria
}

\author{
George I. Ogbu ${ }^{1 *}$, Chris O. Aimakhu², Stephen Ajen Anzaku³, Stephen Ngwan ${ }^{4}$ and Diane A. Ogbu ${ }^{5}$
}

*Correspondence: talktogeorgeogbu@yahoo.com

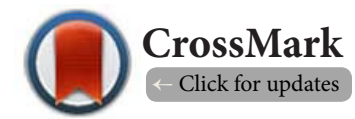

'Department of Obstetrics and Gynaecology, Federal Medical Centre Keffi, Nigeria.

${ }^{2}$ Department of Obstetrics and Gynaecology, University College Hospital Ibadan, Nigeria.

${ }^{3}$ Department of Obstetrics and Gnaecology, College of Medicine and Health sciences, Bingham University, Jos campus, Jos, Nigeria.

${ }^{4}$ Department of Obstetrics and Gynaecology, Federal Medical Centre Makurdi, Nigeria.

${ }^{5}$ Department of computer science, Rehobort Immaculate conception Abuja, Nigeria.

\begin{abstract}
Background: Malaria has been a major public health problem in sub-Saharan Africa. Malaria parasitaemia among pregnant women is associated with adverse maternal and fetal complications. The objective of this study was to determine the prevalence of malaria parasitaemia among asymptomatic women at booking and to ascertain their packed cell volume (PCV) at Garki Hospital, Abuja, Nigeria.

Materials and methods: This was a cross-sectional descriptive study of 659 pregnant women recruited consecutively at the time of booking for antenatal care. Thick film microscopy and thin film for malaria parasites were performed for all the women. PCV assessment was done using the micro centrifuge method and comparison was made between women with and without parasitaemia. Descriptive statistics was also done.

Results: A total of 700 were initially counseled but only 659 gave consent and participated in the study, given a rate of $94 \%$. The gestational age at first booking ranged between 8-37 weeks. Prevalence of malaria parasitaemia was $38.8 \%$. Majority of the women had mild parasitaemia and there was statistical significant difference between mild, moderate and severe parasitaemia. About $53.8 \%$ of primigravidae and $18.7 \%$ multigravidae were anaemic at booking. The Probability of a woman aged $<30$ years having malaria parasitaemia is four times $(81.3 \%)$ more than those aged $>31$ years $(18.8 \%)$. Those with tertiary education constituted the largest group in the Study $(67.2 \%)$ and there was association between low parasitaemia and higher level of education.

Conclusion: Asymptomatic women with malaria parasitaemia and anaemia are common at booking visit. Public enlightenment on malaria prevention and female education may greatly reduce high level of malaria parasitaemia and anaemia among this obstetric population. We recommend screening policy for malaria parasitaemia at booking.
\end{abstract}

Keywords: Malaria parasitaemia, anaemia, asymptomatic women, packed cell volume (PCV)

\section{Introduction}

The importance of malaria in pregnant women and the general population in Sub-saharan Africa cannot be over emphasized. Pregnant women have been shown to have an increased susceptibility to infestation by malaria parasites $[\mathbf{1 , 2 , 4 ]}$. Malaria eradication has eluded most tropical countries and prevention of malaria in pregnancy is a major public health challenge [3]. Each year, tens of thousands of pregnant women in malaria endemic areas like Nigeria are affected by Plasmodium infestations. It is an important public health parasitic infestation in the tropics and tropical Africa bears the greatest burden of the world's malaria [2].

Each year in Sub-sahara Africa where $80-90 \%$ of the world malaria cases occur, approximately 19-24 millions of women are at risk of malaria and its adverse consequences during pregnancy [4-6]. The disease accounts for $40 \%$ of the public 
health expenditure, $50 \%$ of outpatient visits and $30-50 \%$ of inpatient admissions in areas of high transmission (stable malaria transmission) and vast majority of the infestation is with Plasmodium Falciparum $[\mathbf{5 , 7}, \mathbf{1 9}]$ in regions where malaria transmission is stable. Majority of the infestations during pregnancy are said to be asymptomatic, undetected and untreated with attendant major impacts on the mother and the unborn fetus. Women in their first and second pregnancies are most susceptible to Plasmodium Falciparum infestation $[4,8,9]$. Mortality and morbidity from malaria are highest in pregnant women as well as infants and children under the age of five [10,15-17].

Malaria morbidity accounts for $10-80 \%$ of childhood fever, approximately 30 to $35 \%$ of all cases are seen at dispensaries in the Savanna region of Sub Saharan Africa and $10-30 \%$ of all infants and child death in the same region $[11,18]$. Consequently even apparently healthy children in the malaria endemic regions may harbor the parasite in their blood and these children may still suffer the long term sequalae of the disease such as Cognitive impairment and stunting [10,12,13,15-17].

A national Survey conducted in Nigeria in 2000 shows that malaria caused $48.2 \%$ of ailments experienced by pregnant women presenting to the medical practitioner [20]. Thus prevention of malaria in pregnancy is a major public health challenge, an initiative which is encapsulated in the roll back malaria programme $[\mathbf{1 8 , 2 1 , 2 2 ]}$. In order to relieve the economic burden caused by the disease in human, a campaign Roll back malaria (RBM) was initiated in 1998 by the World Health Organization (WHO), United Nations Children's Fund (UNICEF), UNDP and the World Bank with different objectives, one of which intends to halve the burden of malaria by 2010 [23].

Malaria is also a major cause of miscarriages, preterm labor, increased uterine activity, intra uterine fetal death and severe

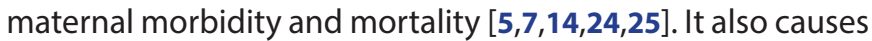
maternal hypoglycaemia, acute pulmonary oedema, cerebral oedema and maternal death $[\mathbf{7 , 1 4 , 2 6 , 2 7 ]}$. Malaria has been responsible directly or indirectly for $10 \%$ of maternal death in Calabar, 8\% in Enugu, 7.8\% in Lagos and 8.2\% in Kano [27]. A recent study from Mozambique had also indicated malaria to be responsible directly for $10 \%$ of maternal death [29].

Although adult living in endemic areas acquire protective immunity against developing severe malaria, they become more susceptible especially when Pregnant $[14,27,29,30,31]$. Generally, a decrease in cell mediated and humoral immunity tends to be more severe in primigravidae than in multigravidae $[25,27,32]$. The resultant effects are more frequent episodes of Plasmodium parasitaemia and greater severity of malaria $[4,27,33]$. In areas with stable malaria like Abuja (Federal Capital Territory), the vast majority of infections with P. Falciparum in Pregnancy remain asymptomatic, undetected and untreated.

The pathogenesis of this severe disease is vast, its effects on the mother and fetus is its massive systematic placental invasion with P. Falciparum. Parasitized erythrocytes are haemolysed and a larger percentage is sequestered into the placental vasculature. The density of malaria parasitaemia in the placenta is about two times the density in the peripheral circulation [2,29,34-36].

Placental Parasitaemia may sometimes exceed $50 \%$ of placental erythrocyte without any parasite in the peripheral blood [2,34-36]. This therefore leads to retro placental hypoxia, inflammatory reactions and chronic intervillositis [35,37-39]. It is to be noted at this junction that the severity of malaria is related to the parasite density rather than clinical presentation $[10,40]$. There is higher parasite density in primigravidae than multigravidae $[2,31,35,36,39]$. There is also increased malaria parasite density in grandmultipara, probably due to progressive loss of immunity in the intervening pregnancies [36].

The importance and severity of malaria in pregnancy has generated extensive debate and research concerning antimalarial Chemoprophylaxis during pregnancy $[18,21,22,41]$. Hence, in 1998 the initiation of the Roll back malaria programme and declaration of African heads of State lead to their commitment to a battle against malaria in April, 2008 [18,21,22,42].

The high level of malaria parasitaemia in the general population especially in a hyperendemic areas and the depression of immunity in pregnancy, call for assessment of the prevalence of malaria parasitaemia at first antenatal visit. This will enhance or lead to a policy of treating all antenatal patients at booking with potent antimalarial drugs before commencement of prophylaxis. This will obviously enhance the prevention of deadly complications of malaria infestation during pregnancy. Hence, this study was undertaken to ascertain the magnitude of the problem as well as anaemic status among asymptomatic pregnant women in our clinical setting.

\section{Materials and methods \\ Study design, population and area}

This was a descriptive cross-sectional study aimed at determining the prevalence of asymptomatic women with malaria infestation during pregnancy at antenatal booking over a two months period at Garki Hospital, Abuja. Garki Hospital is a tertiary health facility involved in the training of undergraduate and postgraduate doctors, situated in Abuja metropolis of the federal capital territory, north central geopolitical zone of Nigeria. It also serves as a referral center for both government and private health care facilities within and outside the country. The vegetation is savanna and has a mean Annual rainfall of $250 \mathrm{~cm}$. Malaria transmission is throughout the year.

\section{Recruitment and data collection}

Informed consent was obtained from the women who came to book for antenatal care within the study period after counseling and were recruited into the study. Women who were symptomatic of malaria were excluded from the study. A prepared structured questionnaire was completed for each participant. Information obtained included age of respondents, 
educational status, occupation, medical history of chronic hypertension, diabetes mellitus, and malaria treatment in the current pregnancy as well as malaria chemoprophylaxis. Obstetrics information included last menstrual period, expected delivery date, gestational age, parity and number of living children.

\section{Laboratory test}

About $2 \mathrm{ml}$ of blood was obtain from a peripheral vein into an ethamine diamine tetraacetic acid (EDTA) bottle for preparation of thick and thin blood film as well as packed cell volume. Two glass slides were labeled for each participant. A drop of blood was then placed on the clean, grease free glass slide and allowed to dry. Precaution was taken to maintain a constant volume as much as possible. The thin smear was made to spread on the glass slide so that newsprint could be read through it. This was immediately fixed in absolute methanol for 5 seconds and allowed to air dry completely before staining. The dried slides were then placed on a rack in preparation for staining. Two capillary tubes were filled with the blood and one end sealed with plasticin gum for each patient for determination of packed cell volume at booking. All those who had anti-malarial or Chemoprophylaxis before booking for antenatal care and gave their consent served as control group.

\section{Packed cell volume estimation}

Using two heparinized capillary tube, $4-5 \mathrm{~cm}$ column of blood was obtained from blood already collected. This was to ensure that the average of the two values obtained is used for calculation. One end of the capillary tube was sealed with plasticin, several samples were assembled in the Centrifuge (haematocrit machine) and spinned at 5000 revolution per minute for 5 minutes. PCV was read using Hawksleys micro haemotocrit reader. Anaemia was diagnosed when packed cell volume was below $33 \%$, according to World health Organization recommendation.

\section{Staining technique for thick blood film}

Thick Blood film was utilized to determine the presence of and quantification of malaria parasites. The thick blood smear was allowed to dry completely under a drier before staining. Giemsa staining technique was used for staining the slides. A staining time of 30 minutes in a $2 \%$ volume/volume dilution was used. The air -dried thick blood film was stained in a trough containing the $2 \%$ giemsa stain for 30 minutes. The slides were then removed with the aid of a forceps, rinsed in buffer water and the back wiped clean with dry wool. The slides were then placed vertically on the staining rack to air-dry before examination.

\section{Staining technique for thin film}

The film staining detects the species of plasmodium. The giemsa staining technique was also used. The thin film already fixed in absolute methanol for 5 seconds was allowed to air dry completely on the staining rack. The slide was then immersed in a trough containing $2 \%$ giemsa for 30 minutes. The stained slides were removed and rinsed in buffer water (PH 7.2). The back side of the slide was wiped with dry cotton wool, kept vertically on the rack to air- dry before examination.

\section{Reading of slides and counting of parasites}

When the slides were completely dried, a drop of oil immersion was placed on each slide and examined using a compound microscope with a $\times 100$ objective magnification. Properly stained areas were selected and observed for malaria parasites. Thick blood film was used and, the method of parasite enumeration was based on WHO approved method $[42,43]$. The number of parasites in a film per 500 white blood cells were counted. At least 500 white blood cells were counted. The number of parasites then were divided by the number of leucocytes and multiplied by a factor of 6000 . This gave the number of parasites per deciliter. The average number of white blood cells (WBC) in blacks per deciliter is 6000 cells.

Parasites count (per $\mathrm{dl}$ ) $=$ number of parasite $X 6000 /$ Number of leucocyte. For the purpose of this study, the following quantification was to describe the densities.

Mild parasitaemia $=<1000$ per dl

Severe parasitaemia $>3000$ per $\mathrm{dl}$

\section{Determination of sample size}

Sample size was determined using the formula $n=Z^{2} p q / d^{2}$ and a prevalence of $54 \%$ reported from a study in Calabar, Nigeria was used [44] at $95 \%$ confidence interval. A sample size of 319 was obtained but 350 women were recruited so as to account for possible attrition.

\section{Data analysis}

These findings were subjected to standard statistical tests using epidemiological information 2002 statistical software of the center for disease control and prevention, Atlanta, USA. Mean were compared using the student t- test and proportions compared using Chi-square test. A p-value of $<0.05$ was considered significant.

\section{Ethical considerations}

Verbal/written informed consent was obtained from each of the participants. Those with high parasite density were recommended for treatment based on the national antimalarial treatment guidelines. Formal approval for the study was obtained from the research ethics committee of the Garki Hospital, Abuja.

\section{Results}

A total of 700 women were initially counseled but only 659 consented and participated in the study, thus giving a rate of participation of $94.1 \%$. The ages of the women ranged between 16-45 years and about two-third of them had tertiary education. 
Ogbu et al. Journal of Reproductive Biology and Health 2015,

Table 1. This shows that the gestational age at booking ranged from $8-37 \%$ weeks. When the gestational age at booking was subjected to Chi-Square analysis, there was statistically significant difference at first booking between the primigravidae and multigravidae. Table 2 shows the association between gravidity, anaemia and parasitaemia. It shows that $53.5 \%$ of primigravidae and $18.7 \%$ of the multigravidae were anaemic at booking. The overall prevalence of anaemia in the study population was $29.4 \%$.

Table 1. Association of gestational age at booking and parasitaemia.

\begin{tabular}{llll}
\hline Gestational age (weeks) & No & Primigravidae & Multigravidae \\
\hline $8-12$ & 101 & $86(22.6 \%)$ & $15(5.4 \%)$ \\
$13-17$ & 74 & $56(14.7 \%)$ & $18(6.5 \%)$ \\
$18-22$ & 202 & $153(40.2 \%)$ & $49(17.6 \%)$ \\
$23-27$ & 114 & $45(11.8 \%)$ & $69(24.8 \%)$ \\
$28-32$ & 152 & $39(10.2 \%)$ & $113(40.6 \%)$ \\
$33-37$ & 16 & $2(0.5 \%)$ & $14(5.0 \%)$ \\
Total & 659 & $381(100 \%)$ & $278(100 \%)$ \\
\hline
\end{tabular}

Chi-square df Probability

$160.8794 \quad 5 \quad 0.0000$

Table 2. Association between gravidity and packed cell volume in parasitaemic women.

\begin{tabular}{llll}
\hline $\begin{array}{l}\text { Packed cell } \\
\text { volume }\end{array}$ & No & Primigravidae & Multigravidae \\
\hline$<33$ & $256(38.8 \%)$ & $204(53.5 \%)$ & $52(18.7 \%)$ \\
$\geq 33$ & $403(61.2 \%)$ & $177(43.9 \%)$ & $226(81.3 \%)$ \\
Total & $659(100.0 \%)$ & $381(100 \%)$ & $278(100 \%)$ \\
\hline
\end{tabular}

Table 3 shows the presence of malaria parasitaemia among the study population. Among women who had no antimalarial, one hundred and ninety four (55.4\%) of them had parasitaemia While one hundred and fifty six (44.6\%) had no parasite in their blood. However, those who had antimalarial and still had parasitaemia were sixty two $(20.1 \%)$ as against two hundred and forty seven (79.9\%) of them with no parasite in their blood. There was a statistically significant difference between the two groups. The overall prevalence of malaria parasitaemia among pregnant women at first antenatal visit was $38.8 \%(256 / 659)$.

Table 4 shows the severity of parasitaemia densities. A total of three hundred and fifty women had no antimalarial treatment and out of this, $44.6 \%$ had no parasites, $34.0 \%$ had mild parasitaemia, $18.3 \%$ had moderate parasitaemia and $3.1 \%$ had severe parasitaemia. Three hundred and nine women had antimalarial treatment and out of this, $80.3 \%$ had no parasites, $18.4 \%$ had mild parasitaemia, while $1.3 \%$ had moderate parasite density. Table 5 shows the association between severity of parasitaemia and anaemia. A total of four
Table 3. Gross presence of malaria parasitaemia.

\begin{tabular}{llll}
\hline Parasitaemia & Had no antimalarial & Had antimalarial & Total \\
\hline Yes & $194(55.4 \%)$ & $62(20.1 \%)$ & 256 \\
No & $156(44.6 \%)$ & $247(79.9 \%)$ & 403 \\
Total & $350(100.0 \%)$ & $309(100 \%)$ & 659 \\
\hline
\end{tabular}

Odd's ratio Risk ratio Chi-square

$0.2018 \quad 0.5108 \quad 86.3945$

Table 4. Parasite count/severity.

\begin{tabular}{lll}
\hline $\begin{array}{l}\text { Severity of } \\
\text { parasitaemia }\end{array}$ & Had no antimalarial & Had antimalarial \\
\hline No parasite & $156(44.6 \%)$ & $248(80.3 \%)$ \\
Mild & $119(34.0 \%)$ & $57(18.4 \%)$ \\
Moderate & $64(18.3 \%)$ & $4(1.3 \%)$ \\
Severe & $11(3.1 \%)$ & $0(0.0 \%)$ \\
Total & $350(100.0 \%)$ & $309(100 \%)$ \\
\hline
\end{tabular}

Chi-square df Probability

$104.5866 \quad 3 \quad 0.0000$

hundred and six women had no anaemia, (packed cell volume of $33 \%$ ) and out of this $77.3 \%$ had no parasite in their red blood cells, $17.5 \%$ had mild parasitaemia, $4.7 \%$ had moderate parasitaemia and $0.5 \%$ had severe parasitaemia. Women with packed cell volume below 33 (anaemia) were two hundred and fifty three and out of this $35.6 \%$ had no parasite in their blood, $41.5 \%$ had mild parasitaemia, $19.4 \%$ had moderate parasitaemia and only $3.6 \%$ had severe parasitaemia. When the association between parasitaemia and anaemia was compared it was statistically significant $(P<0.00001)$.

\section{Discussion}

The age of the subjects in the study was between 16-45 years which is similar to earlier finding in Kano, Nigeria [27]. The prevalence of malaria parasitaemia is usually higher in the rural areas where mosquito breeding and transmission is intense. The fact that about two-third of the study population had tertiary education may have contributed to the high participation in this study. High standard of education usually affect health awareness and therefore has a positive impact on health [4]. This may also have contributed to the low level of parasitaemia obtained in this study since they were probably better informed about vector control such as the use of insecticide treated nets [4]. Ilobachie et al., [44] in Enugu South eastern Nigeria reported positive influence of formal education on the use of maternity services. Some of the women took antimalarial since they were learned and knew some of the antimalarial available over the counter in Nigeria. This may explain why this group of women had lower parasitaemia as noted in this study. This is corroborated by the finding by Gajida et al., [27] who reported that self-treatment of malaria led to a reduction in prevalence of parasitaemia 
Table 5. Associations between severity of parasitaemia and anaemia.

\begin{tabular}{llllll}
\hline Packed cell volume \% & No parasite seen & Mild<1000/DL & Moderate 1000-2999/DL & Severe>3000/DL & Total \\
\hline $21-32$ & $90(35.6 \%)$ & $105(41.5 \%)$ & $49(19.4 \%)$ & $9(3.6 \%)$ & $253(100.0 \%)$ \\
$\geq 33$ & $314(77.3 \%)$ & $71(17.5 \%)$ & $19(4.7 \%)$ & $2(0.5 \%)$ & $406(100.0 \%)$ \\
Total & $404(61.3 \%)$ & $176(26.7 \%)$ & $68(10.3 \%)$ & $11(1.7 \%)$ & $659(100.0 \%)$ \\
\hline
\end{tabular}

Chi-square df Probability

$\begin{array}{lll}119.3683 & 3 & 0.0000\end{array}$

$\mathrm{P}<0.05$, therefore, there is a significant relationship between parasitaemia and anaemia.

among pregnant women attending antenatal care. However it is contrary to finding by Arnolu et al., [45] who reported that previous use of antimalarial drug before booking had no significant effect on the prevalence of malaria parasitaemia in a study conducted in Lagos.

Anaemia was significantly associated with malaria parasitaemia in this study which is also similar to other studies $[4,27,33]$. The prevalence of anaemia among patients with parasitaemia was $38.85 \%$. Despite the presence of anaemia in these women, they were asymptomatic for malaria. Although the gestational age at booking among primigravidae was significantly lower than among multigravidae and this pattern of late booking during the second and third trimester is in keeping with a previous report [47]. This late antenatal booking is detrimental to the achievement of safe motherhood in this group of women.

The prevalence of malaria parasitaemia was highest in the second trimester because most of the women book at this stage of pregnancy which is in keeping with a previous report [47] and as such, some of the clients may have the opportunity of getting the first and second dose of antimalarial prophylaxis. The prevalence of anaemia at first antenatal visit in this study was lower than figures from other studies $[4,27,47]$. This may be due to the fact that most of the women were educated and hence a probable better nutritional status. Also it may be explained by the fact that the work was conducted in an urban Nigerian city with good malaria vector control.

Parasite density in this study had influence on the severity of anaemia. Higher parasite density had more effect on the packed cell volume with more severe degree of anaemia. It is important to note that asymptomatic malaria parasitaemia is one of the major causes of anaemia in our malaria hyper endemic environment. Apart from the reduced immunity which is marked in the first pregnancy, the most important influence of the infestation on maternal health is caused by anaemia. A higher proportion of those with malarial parasitaemia were anaemic compared with those without malarial parasitaemia. Denser malaria parasitaemia lead to increased red blood cell haemolysis ultimately leading to anaemia, which is usually normochromic and normocytic and accompany by reticulocytosis. Other effects of malaria in pregnancy include abortion, preterm labour, intra uterine fetal death, puerperal pyrexia and low birth weight.
Participants who did not have any form of antimalarial drugs before booking and had parasitaemia were $55.4 \%$ of the study population while those that took antimalarial and had parasitaemia were $20.1 \%$. This suggest that antimalarial at any dose had significant effect on parasitaemia. High parasite density is of public health importance in pregnancy. This is associated with red blood cell haemolysis with resultant anaemia in pregnancy and fetal complications.

A total of $55.4 \%$ Primigravidae and $20.1 \%$ multigravidae were parasitaemic at booking but a general prevalence of $38.8 \%$. This prevalence is similar to that of the work done in Jos by Egwunyenga et al., and the reports by Gajida [27] and Agboghohoroma [48]. It is however, lower than findings from other researchers $[4,25,47]$. Majority of the women with malaria parasitaemia (81.3\%) were aged 30 years and below while $18.8 \%$ were aged above 30 years which is in agreement with previous findings that the severity of parasitaemia tends to be higher in younger pregnant women.

There was statistically significant association between parasitaemia and the use of malaria chemoprophylaxis and treatment. The proportions of women who took antimalarial drugs and still had parasitaemia were far less than those who had malaria drugs and were not parasitaemic. There was also statistically significant association between malaria chemoprophylaxis and treatment with the high prevalence of anaemia in this study, which suggests that not all malaria drugs may be effective or potent.

\section{Conclusion}

Malaria in pregnancy is a common and serious public health problem in our environment as large proportion of the asymptomatic pregnant women had malaria parasitaemia. Malarial infestation during pregnancy affect more primigravidae and teenage mothers than those of higher gravidity and older age group. Anaemia is also a serious problem especially among pregnant women with asymptomatic parasitaemia. Therefore this study has indicated that malaria is a contributor to anaemia among these pregnant women.

\section{Recommendations}

We recommend public enlightenment on malaria among women and girl child education so as to reduce the proportion of women who present for antenatal booking with parasitaemia 
and anaemia. There should also be a Federal Government policy to aimed at screening all pregnant women for malaria parasitaemia and anaemia especially primigravidae at booking so that appropriate antimalarial therapy is instituted to clear the parasitaemia and this will lead to reduction in level of anaemia. There is a need for a comprehensive strategy including intermittent preventive treatment of malaria in pregnancy, good nutrition and effective use of insecticide treated bed nets among pregnant women in this endemic region.

\section{Competing interests}

The authors declare that they have no competing interests. Authors' contributions

\begin{tabular}{|l|c|c|c|c|c|}
\hline Authors' contributions & GIO & COA & SAA & SN & DAO \\
\hline Research concept and design & $\checkmark$ & $\checkmark$ & $\checkmark$ & -- & -- \\
\hline Collection and/or assembly of data & $\checkmark$ & -- & -- & -- & -- \\
\hline Data analysis and interpretation & $\checkmark$ & $\checkmark$ & $\checkmark$ & -- & -- \\
\hline Writing the article & $\checkmark$ & $\checkmark$ & $\checkmark$ & -- & $\checkmark$ \\
\hline Critical revision of the article & $\checkmark$ & $\checkmark$ & $\checkmark$ & $\checkmark$ & $\checkmark$ \\
\hline Final approval of article & $\checkmark$ & $\checkmark$ & $\checkmark$ & $\checkmark$ & $\checkmark$ \\
\hline Statistical analysis & $\checkmark$ & -- & -- & -- & -- \\
\hline
\end{tabular}

\section{Acknowledgement}

We wish to acknowledge the contributions of the laboratory scientists in the hospital for their assistance in samples collection and analysis in the laboratory.

\section{Publication history}

Editor: Erich Cosmi, University of Padua, Italy.

EIC: Zeev Blumenfeld, Technion-Israel Institute of Technology, Israel.

Received: 02-Feb-2015 Final Revised: 02-Mar-2015

Accepted: 27-Mar-2015 Published: 03-Apr-2015

\section{References}

1. Ter Kuile FO, Terlouw DJ, Phillips-Howard PA, Hawley WA, Friedman JF, Kariuki SK, Shi YP, Kolczak MS, Lal AA, Vulule JM and Nahlen BL. Reduction of malaria during pregnancy by permethrin-treated bed nets in an area of intense perennial malaria transmission in western Kenya. Am J Trop Med Hyg. 2003; 68:50-60. | Article | PubMed

2. World Health Organization. A Strategic frame work for malaria prevention and control during pregnancy in the African region. Brazzaville 2004. | Article

3. Nyarango PM, Gebremeskel T, Mebrahtu G, Mufunda J, Abdulmumini U, Ogbamariam A, Kosia A, Gebremichael A, Gunawardena D, Ghebrat Y and Okbaldet $Y$. A steep decline of malaria morbidity and mortality trends in Eritrea between 2000 and 2004: the effect of combination of control methods. Malar J. 2006; 5:33. | Article | PubMed Abstract | PubMed Full $\underline{\text { Text }}$

4. Isah AY, Amanabo MA and Ekele BA. Prevalence of malaria parasitemia amongst asymptomatic pregnant women attending a Nigerian teaching hospital. Ann Afr Med. 2011; 10:171-4. | Article | PubMed

5. WHO. Malaria in Africa. Roll back malaria info sheet. 2007. | Website

6. Guyatt $\mathrm{HL}$ and Snow RW. The epidemiology and burden of Plasmodium falciparum-related anemia among pregnant women in sub-Saharan Africa. Am J Trop Med Hyg. 2001; 64:36-44. | PubMed

7. WHO. Malaria in pregnancy. Roll Back malaria info sheet. 2007.

8. Desowitz RS and Alpers MP. Placental Plasmodium falciparum parasitaemia in East Sepik (Papua New Guinea) women of different parity: the apparent absence of acute effects on mother and foetus. Ann Trop Med Parasitol. 1992; 86:95-102. | PubMed
9. Bouyou-Akotet MK, Issifou S, Meye JF, Kombila M, Ngou-Milama E, Luty AJ, Kremsner PG and Mavoungou E. Depressed natural killer cell cytotoxicity against Plasmodium falciparum-infected erythrocytes during first pregnancies. Clin Infect Dis. 2004; 38:342-7. | Article | PubMed

10. Patrick Kachur S, Schulden J, Goodman CA, Kassala H, Elling BF, Khatib RA, Causer LM, Mkikima S, Abdulla S and Bloland PB. Prevalence of malaria parasitemia among clients seeking treatment for fever or malaria at drug stores in rural Tanzania 2004. Trop Med Int Health. 2006; 11:44151. | Article | PubMed

11. Centers for disease control and prevention. The impact of malaria, a leading cause of death worldwide. Malaria.

12. Custodio E, Descalzo MA, Villamor E, Molina L, Sanchez I, Lwanga M, Bernis C, Benito A and Roche J. Nutritional and socio-economic factors associated with Plasmodium falciparum infection in children from Equatorial Guinea: results from a nationally representative survey. Malar J. 2009; 8:225. | Article | PubMed Abstract | PubMed Full Text

13. Ikeh El and Teclaire NN. Prevalence of malaria parasitaemia and associated factors in febrile under- 5 children seen in Primary Health Care Centres in Jos, North Central Nigeria. Niger Postgrad Med J. 2008; 15:65-9. | PubMed

14. WHO. Lives at risk: Malaria in pregnancy. 2007.

15. Yartey JE. Malaria in pregnancy: access to effective interventions in Africa. Int J Gynaecol Obstet. 2006; 94:364-73. | Article | PubMed

16. Ashley E, McGready R, Proux S and Nosten F. Malaria. Travel Med Infect Dis. 2006; 4:159-73. | Article | PubMed

17. Sarr D, Marrama L, Gaye A, Dangou JM, Niang M, Mercereau-Puijalon $\mathrm{O}$, Lehesran JY and Jambou R. High prevalence of placental malaria and low birth weight in Sahelian periurban area. Am J Trop Med Hyg. 2006; 75:171-7. | Article | PubMed

18. Daboer JC, Chingle MP and Ogbonna C. Malaria parasitaemia and Household use of insecticide treated bed Nets; A cross- sectional Survey of under-fives in Jos, Nigeria. Niger med J. 2010; 51. | Article

19. Idowu ET, Mafe MA, Otubanjo OA and Adeneye AK. Herbal remedy in the treatment of malaria: cross sectional survey of residents of Lagos State, Nigeria. Afr J Med Med Sci. 2006; 35:149-53. | PubMed

20. Federal ministry of Health, Nigeria: National Survey malaria 2000.

21. World Health organization. World malaria Report 2005. Geneva.RBM/ WHO/UNICEF 2005.

22. Mathanga DP, Campbell CH, Taylor TE, Barlow R and Wilson ML. Reduction of childhood malaria by social marketing of insecticidetreated nets: a case-control study of effectiveness in Malawi. Am J Trop Med Hyg. 2005; 73:622-5. | Article | PubMed

23. Agbaje EO and Aphia DO. Knowledge and management of malaria among non-medical Nigerian undergraduates. Niger Postgrad Med J. 2007; 14:221-5. | PubMed

24. Onah HE, NKwo PO and Nwankwo TO. Malaria chemoprophylaxis during pregnancy: A Survey of current practice amongst Nigerian Obstetricians. Trop J ObstetGynaecol. 2006; 23:17-19. | Article

25. Falade CO, Olayemi O, Dada-Adegbola HO, Aimakhu CO, Ademowo $\mathrm{OG}$ and Salako LA. Prevalence of malaria at booking among antenatal clients in a secondary health care facility in Ibadan, Nigeria. Afr J Reprod Health. 2008; 12:141-52. | PubMed

26. Federal ministry of Health. Malaria situation analysis. 2000.

27. Gajida AU, Iliyasu Z and Zoakah AI. Malaria among antenatal clients attending primary health care facilities in Kano state, Nigeria. Ann Afr Med. 2010; 9:188-93. | Article | PubMed

28. Society for Gynaecology and Obstetrics of Nigeria (SOGON). Report of the maternal mortality situation in six(6) tertiary hospitals in Nigeria. Needs assessment. 2004; 1-65.

29. Schantz-Dunn J and Nour NM. Malaria and pregnancy: a global health perspective. Rev Obstet Gynecol. 2009; 2:186-92. | PubMed Abstract | PubMed Full Text

30. Greenwood BM, Fidock DA, Kyle DE, Kappe SH, Alonso PL, Collins FH and Duffy PE. Malaria: progress, perils, and prospects for eradication. J Clin Invest. 2008; 118:1266-76. | Article | PubMed Abstract | PubMed Full Text 
Ogbu et al. Journal of Reproductive Biology and Health 2015,

http://www.hoajonline.com/journals/pdf/2054-0841-3-1.pdf

31. Ofori M, Ansah E, Agyepong I, Ofori-Adjei D, Hviid L and Akanmori B. Pregnancy-associated malaria in a rural community of ghana. Ghana Med J. 2009; 43:13-8. | PubMed Abstract | PubMed Full Text

32. Okafor UH, Oguonu T and Onah HE. Risk factors associated with congenital malaria in Enugu, South Eastern Nigeria. J Obstet Gynaecol. 2006; 26:612-6. | Article | PubMed

33. Idowu OA, Mafiana CF and Dapo S. Malaria among pregnant women in Abeokuta, Nigeria. Tanzan Health Res Bull. 2006; 8:28-31. | Article | PubMed

34. Akinboro RA, Ojurongbe $O$, Akindele AA, Adefioye $O A$, Bolajios and Olaniran $O$. Plasmodium Falciparum parasitaemia in pregnancy in relation to maternal Anaemia. Afr J ClinEperMicrobiol. 2010; 11:164169. | PubMed

35. Federal Ministry of Health, Nigeria. Malaria control in Nigeria: A strategy for Behaviour change communication 2004: 5-59.

36. Federal Ministry of health, Nigeria. National Guide lines and Strategies for Malaria prevention and control during pregnancy 2004; 1-31.

37. White NJ, Breman JG. Malaria.In: Fauci AS, Braunwald E and Kasper DL et al. eds. Harrison's principles of Internal medicine. 17th ed. New York: mc Graw-Hill; 2008: 1280-1293.

38. Opare- Addo HS, Odio AT. Malaria in pregnancy. In: KwawukumeEY, Emuveyan EE (Eds). Comprehensive Obstetrics in the tropics. First Edition. Asante \&\&Hittscher printing press Limited 2002: 250-260.

39. Shulman CE and Dorman EK. Importance and prevention of malaria in pregnancy. Trans R Soc Trop Med Hyg. 2003; 97:30-5. | Article | PubMed

40. Smith DL, Guerra CA, Snow RW and Hay SI. Standardizing estimates of the Plasmodium falciparum parasite rate. Malar J. 2007; 6:131. | Article | PubMed Abstract | PubMed Full Text

41. Senbanjo IO, Adeodu OO, Ogunlesi TA, Anyabolu CH and Okusanya AA. The use of antimalaria drugs and insecticide treated nets in lle-Ife, Nigeria. Niger J Med. 2006; 15:277-80. | PubMed

42. World Health Organization (WHO) Secretariat for coordination of malaria training in Asia and the Specific bench Aids for the diagnostic of malaria. 1985; $1: 4$.

43. Wufo CA. Introduction to medical Statistics Research Methodology. Wueli publishing Services, Accra 2003:257-264.

44. Iloabechie GC and Meniru G. I Incidence of anaemia in pregnancy in Nigeria. Orient journal of medicine. 1990; 2: 194-198.

45. Anorlu RI, Odum CU and Essien EE. Asymptomatic malaria parasitaemia in pregnant women at booking in a primary health care facility in a periurban community in Lagos, Nigeria. Afr J Med Med Sci. 2001; 30 Suppl:39-41. | PubMed

46. Agan TU, Ekabua JE, Udoh AE, Elkanem El Effiok EE and Mgbekem MA. Prevalence of anaemia in women with asymptomatic malaria parasitaemia at first antenatal care visit at the University of Calabar Teaching Hospital, Calabar, Nigeria. Int J. women health. 2010; 2:229233. | Article

47. Egwunyenya Ao, Ajayi JA, Mnorsi OP and Duhhinksla-popoola DD. Plasmodum/intestinal helminth co infection among pregnant Nigeria. women merinstoswaldocruz. 2001; 96:1055-9.

48. Agboghoroma OC, ElagbaOY, Ladipo OF, Umezeurike AC,, Efetie RE and Tabarisi S. Prevalence of asymptomatic malariaparasitaemia in pregnant women at first antenatal visit in Abuja. Book of abstract of the 38 annual scientific conference of the society of obstetrics and gynaecology of Nigeria (SOGON). Makurdi 2002; 21-536.

\section{Citation:}

Ogbu GI, Aimakhu CO, Ajen Anzaku S, Ngwan S and Ogbu DA. Prevalence of malaria parasitaemia among asymptomatic women at booking visit in a tertiary hospital, Northcentral Nigeria. J Reprod Biol Health. 2015; 3:1. http://dx.doi.org/10.7243/2054-0841-3-1 